\title{
Solvability of Some Two-Point Fractional Boundary Value Problems under Barrier Strip Conditions
}

\author{
Limei He, ${ }^{1}$ Xiaoyu Dong, ${ }^{1}$ Zhanbing Bai, ${ }^{1}$ and Bo Chen ${ }^{2}$ \\ ${ }^{1}$ College of Mathematics and System Science, Shandong University of Science and Technology, Qingdao 266590, China \\ ${ }^{2}$ College of Mathematics and Statistics, Shenzhen University, Shenzhen 518060, China \\ Correspondence should be addressed to Zhanbing Bai; zhanbingbai@163.com
}

Received 14 July 2017; Accepted 27 September 2017; Published 26 October 2017

Academic Editor: Manuel De la Sen

Copyright (c) 2017 Limei He et al. This is an open access article distributed under the Creative Commons Attribution License, which permits unrestricted use, distribution, and reproduction in any medium, provided the original work is properly cited.

Topological techniques are used to establish existence results for a class of fractional differential equations $D^{\alpha} x(t)=$ $f\left(t, x(t), D^{\alpha-1} x(t)\right)$, with one of the following boundary value conditions: $x(0)=A$ and $D^{\alpha-1} x(1)=B$ or $D^{\alpha-1} x(0)=A$ and $x(1)=B$, where $1<\alpha \leq 2$ is a real number, $D^{\alpha} x(t)$ is the conformable fractional derivative, and $f:[0,1] \times R^{2} \rightarrow R$ is continuous. The main conditions on the nonlinear term $f$ are sign conditions (i.e., the barrier strip conditions). The topological arguments are based on the topological transversality theorem.

\section{Introduction}

Recently, boundary value problems of nonlinear fractional differential equations have been addressed by several researchers. Fractional differential equations arise in many engineering and scientific disciplines as the mathematical modeling of systems and processes in the fields of physics, chemistry, control theory, biology, economics, blood flow phenomena, signal and image processing, biophysics, aerodynamics, fitting of experimental data, and so forth. For example, in 2006 , by using the fixed point theorem in cones, the existence and multiplicity of solutions to the following problems are obtained [1]:

$$
\begin{aligned}
{ }^{\mathscr{C}} D_{0+}^{\alpha} u(t) & =f(t, u(t)), \\
u(0)+u^{\prime}(0) & =0, \\
u(1)+u^{\prime}(1) & =0,
\end{aligned}
$$

where $1<\alpha \leq 2$ is a real number, ${ }^{\mathscr{C}} D_{0+}^{\alpha} u(t)$ is Caputo fractional derivative, and $f:[0,1] \times[0, \infty) \rightarrow[0, \infty)$ is a continuous function. In 2010, by the use of the Lipschitz condition and the compression mapping principle, the existence of solutions of the following problem is obtained [2]:

$$
\begin{aligned}
D_{0+}^{\alpha} u(t)+f(t, u(t)) & =0, \\
u(0) & =0, \\
\beta u(\eta) & =u(1),
\end{aligned}
$$

where $1<\alpha \leq 2$ is a real number, $0<\beta \eta^{\alpha-1}<1,0<\eta<1$, $D_{0+}^{\alpha} u(t)$ is the standard Riemann-Liouville derivative, and $f$ : $[0,1] \times[0, \infty) \rightarrow[0, \infty)$ is a continuous function. We refer the readers to other contributions in this line ([1-30], etc.).

The technique of barrier strips has been used by Kelevedjiev and Tersian in [31,32] to study the solvability of integer order BVPs. Recently, we use it to study the fractional differential equation with Dirichlet boundary value condition [20]. In this paper, by using the topological transversality theorem, we consider the following equation:

$$
D^{\alpha} x(t)=f\left(t, x(t), D^{\alpha-1} x(t)\right),
$$

with one of the following boundary value conditions:

$$
\begin{aligned}
x(0) & =A, \\
D^{\alpha-1} x(1) & =B ; \text { or } \\
D^{\alpha-1} x(0) & =A, \\
x(1) & =B,
\end{aligned}
$$


where $1<\alpha \leqslant 2$ is a real number, $D^{\alpha} x(t)$ is the conformable fractional order derivative, and $f:[0,1] \times R^{2} \rightarrow R$ is a continuous function. The existence results of solutions to the problem are obtained under $f$ which satisfies some barrier strip conditions.

\section{Definitions and Lemmas about Fractional Calculus}

Let $\alpha \in(n, n+1)$.

Definition 1 (see [14]). Suppose $u$ is $n$-order differentiable for $t \geq 0$; the $\alpha$-order fractional derivative of $u$ is defined as

$$
D^{\alpha} u(t)=\lim _{\varepsilon \rightarrow 0} \frac{u^{(n)}\left(t+\varepsilon t^{n+1-\alpha}\right)-u^{(n)}(t)}{\varepsilon},
$$

provided the limits of the right side exist.

Lemma 2 (see [12]). Let $t>0$. Function $u(t)$ is $\alpha$-order differentiable if and only if $u$ is $(n+1)$-order differentiable; moreover, the following relation holds:

$$
D^{\alpha} u(t)=t^{n+1-\alpha} u^{(n+1)}(t) .
$$

Definition 3 (see [14]). The $\alpha$-order fractional integral is defined as

$$
\begin{aligned}
J_{0+}^{\alpha} u(t) & =I^{n+1}\left[t^{\alpha-n-1} u(t)\right] \\
& =\frac{1}{n !} \int_{0}^{t}(t-s)^{n} s^{\alpha-n-1} u(s) d s,
\end{aligned}
$$

where $I^{n+1}$ is the $(n+1)$-order integral.

Lemma 4 (see $[20])$. For $i=0,1, \ldots, n$, there holds

$$
\begin{aligned}
D^{\alpha-i}\left[J_{0+}^{\alpha} u(t)\right] & =t^{n+1-\alpha} D^{n+1-i}\left[I^{n+1}\left(t^{\alpha-n-1} u(t)\right)\right] \\
& =t^{n+1-\alpha} I^{i}\left[t^{\alpha-n-1} u(t)\right] .
\end{aligned}
$$

Lemma 5 (see [14]). Let $a \geq 0, f:[a, b] \rightarrow R$ be continuous on $[a, b]$ and $\alpha$-order differentiable on $(a, b)$. Then, there exists $d \in(a, b)$ such that

$$
f(b)-f(a)=D^{\alpha} f(d) \frac{b^{\alpha}-a^{\alpha}}{\alpha} .
$$

\section{Topological Preliminaries and a New Function Space}

We begin with a brief review of the topological results to be used in this paper; see [33]. Let $C$ be a convex subset of a Banach space $E, X$ a metric space, and $F: X \rightarrow C$ a continuous map. We say that $F$ is compact if $F(X)$ is contained in a compact subset of $C$. $F$ is completely continuous if it maps bounded subsets in $X$ into compact subsets of $C$. A homotopy $\left\{H_{t}: X \rightarrow C\right\}_{0 \leq t \leq 1}$ is said to be compact provided that $H: X \times[0,1] \rightarrow C$ given by $H(x, t)=H_{t}(x)$ for $(x, t)$ in $X \times[0,1]$ is compact.
Let $U \subset C$ be open in $C$. A compact map $F: \bar{U} \rightarrow C$ is called admissible if it is fixed point free on the boundary, $\partial U$, of $U$. The set of all such maps will be denoted by $\aleph_{\partial U}(\bar{U}, C)$.

Definition 6. A map $F$ in $\aleph_{\partial U}(\bar{U}, C)$ is inessential if there is a fixed point free compact map $G: \bar{U} \rightarrow C$ such that $G \mid \partial U=$ $F \mid \partial U$. A map $F$ in $\aleph_{\partial U}(\bar{U}, C)$ which is not inessential is called essential.

Lemma 7 (see [33]). Let $p$ be an arbitrary value in $U$ and $F$ be in $\aleph_{\partial U}(\bar{U}, C)$ and be the constant map $F(x) \equiv p$ for $x$ in $\bar{U}$. Then $F$ is essential.

Definition 8. Two maps $F$ and $G$ in $\aleph_{\partial U}(\bar{U}, C)$ are called homotopic $(F \sim G)$ if there is a compact homotopy $H_{t}: \bar{U} \rightarrow$ $C$ such that $F=H_{0}$ and $G=H_{1}$ and $H_{t}$ is admissible for each $t$ in $[0,1]$.

The following theorem called topological transversality theorem which is very important to our results.

Lemma 9 (see [33]). Let $F$ and $G$ be in $\aleph_{\partial U}(\bar{U}, C)$ and be homotopic maps, $F \sim G$. Then one of these maps is essential if and only if the other is.

Now, we construct a function space. Given $\alpha>0$, let $\alpha \epsilon$ $(n, n+1]$. Define

$$
\begin{array}{r}
C^{\alpha}[0,1]=\left\{u \mid u(t)=J_{0^{+}}^{\alpha} x(t)+C_{n} t^{n}+\cdots+C_{1} t\right. \\
\left.\quad+C_{0}, C_{i} \in R, i=0,1, \ldots, n, x(t) \in C[0,1]\right\} .
\end{array}
$$

By the linearity of integral operator $J_{0^{+}}^{\alpha}, C^{\alpha}[0,1]$ is a linear space. For $u \in C^{\alpha}[0,1]$, according to Lemma 4 , there are $D^{\alpha-i} u(t) \in C[0,1], i=0,1, \ldots, n$. Define

$$
\|u\|_{\alpha}=\left\|D^{\alpha} u\right\|_{0}+\left\|D^{\alpha-1} u\right\|_{0}+\cdots+\left\|D^{\alpha-n} u\right\|_{0}+\|u\|_{0},
$$

where $\|u\|_{0}=\max _{t \in[0,1]}|u(t)|$. Next, we prove $\|\cdot\|_{\alpha}$ is a norm in the linear space $C^{\alpha}[0,1]$, and $C^{\alpha}[0,1]$ is complete with this norm.

Theorem 10. $\left(C^{\alpha}[0,1],\|\cdot\|_{\alpha}\right)$ is a Banach space.

Proof. It is easy to verify that $\|\cdot\|_{\alpha}$ satisfies the norm axioms. The following proof is about the completeness of $C^{\alpha}[0,1]$.

Let $\left\{u_{k}\right\}_{k=1}^{\infty} \subset C^{\alpha}[0,1]$ be a Cauchy sequence in $C^{\alpha}[0,1]$ :

$$
u_{k}(t)=J_{0^{+}}^{\alpha} y_{k}(t)+C_{k, n} t^{n}+\cdots+C_{k, 1} t+C_{k, 0},
$$

where $y_{k}(t) \in C[0,1]$ and $C_{i, j} \in R(i=1,2, \ldots, k ; j=$ $0,1, \ldots, n)$. Then

$$
\begin{aligned}
u_{k}(t)-u_{m}(t)= & J_{0^{+}}^{\alpha}\left(y_{k}(t)-y_{m}(t)\right) \\
& +\left(C_{k, n}-C_{m, n}\right) t^{n}+\cdots \\
& +\left(C_{k, 1}-C_{m, 1}\right) t+\left(C_{k, 0}-C_{m, 0}\right) .
\end{aligned}
$$


Because $\left\{u_{k}\right\}$ is a Cauchy sequence of the Banach space $C^{\alpha}[0,1]$,

$$
\begin{aligned}
\left\|u_{k}-u_{m}\right\|_{\alpha}= & \left\|D^{\alpha}\left(u_{k}-u_{m}\right)\right\|_{0}+\left\|D^{\alpha-1}\left(u_{k}-u_{m}\right)\right\|_{0} \\
& +\cdots+\left\|D^{\alpha-n}\left(u_{k}-u_{m}\right)\right\|_{0} \\
& +\left\|\left(u_{k}-u_{m}\right)\right\|_{0} \longrightarrow 0, \\
& \text { when } k, m \longrightarrow \infty .
\end{aligned}
$$

Thus every term of the above formula converges to 0 . By

$$
\begin{aligned}
\left\|D^{\alpha}\left(u_{k}-u_{m}\right)\right\|_{0}=\left\|y_{k}-y_{m}\right\|_{0} \longrightarrow & \\
& \text { as } k, m \longrightarrow \infty,
\end{aligned}
$$

we know $\left\{y_{k}\right\}_{k=1}^{\infty}$ is a Cauchy sequence in $C[0,1]$. By the completeness of $C[0,1]$, there exists $y \in C[0,1]$ such that $y_{k} \rightarrow y$. The second term is

$$
\begin{aligned}
& \left\|D^{\alpha-1}\left(u_{k}-u_{m}\right)\right\|_{0}=\| D^{\alpha-1} J_{0^{+}}^{\alpha}\left(y_{k}-y_{m}\right)+\left(C_{k, n}\right. \\
& \left.-C_{m, n}\right) D^{\alpha-1} t^{n} \|_{0} \\
& =\| t^{n+1-\alpha} \int_{0}^{t} s^{\alpha-n-1}\left[y_{k}(s)-y_{m}(s)\right] d s \\
& +\left(C_{k, n}-C_{m, n}\right)\left(n ! \cdot t^{n+1-\alpha}\right) \|_{0} \longrightarrow 0, \\
& \quad \text { as } k, m \rightarrow \infty .
\end{aligned}
$$

Taking into account that $\left\{y_{k}\right\}$ is a Cauchy sequence in $C[0,1]$, there is

$$
\begin{aligned}
& \left\|\left(C_{k, n}-C_{m, n}\right)\left(n ! \cdot t^{n+1-\alpha}\right)\right\|_{0} \\
& \leq \| t^{n+1-\alpha} \int_{0}^{t} s^{\alpha-n-1}\left[y_{k}(s)-y_{m}(s)\right] d s \\
& +\left(C_{k, n}-C_{m, n}\right)\left(n ! \cdot t^{n+1-\alpha}\right) \|_{0} \\
& +\left\|t^{n+1-\alpha} \int_{0}^{t} s^{\alpha-n-1}\left[y_{k}(s)-y_{m}(s)\right] d s\right\|_{0} \longrightarrow 0 .
\end{aligned}
$$

That is to say, $\left\{C_{k, n}\right\}_{k=1}^{\infty}$ is a Cauchy sequence in $R$. Denote its limit as $C_{n}$. Continue this process, and we can prove that sequences $\left\{C_{k, i}\right\}_{k=1}^{\infty}, i=0,1, \ldots, n$, all are Cauchy sequences. Denote their limits as $C_{n-1}, C_{n-2}, \ldots, C_{1}, C_{0}$, respectively.

Let $u(t)=J_{0^{+}}^{\alpha} y(t)+C_{n} t^{n}+\cdots+C_{1} t+C_{0}$, and then $u(t) \epsilon$ $C^{\alpha}[0,1]$ and $\left\|u_{k}-u\right\|_{\alpha} \rightarrow 0,(k \rightarrow \infty)$. The completeness of $C^{\alpha}[0,1]$ is proved.

\section{Main Results}

Denote

$$
\begin{aligned}
G_{1} & =\max \left\{\left|L_{1}\right|,\left|L_{4}\right|\right\}, \\
G_{2} & =|A|+\frac{G_{1}}{(\alpha-1)}, \\
G_{3} & =\max _{[0,1] \times\left[-G_{2}, G_{2}\right] \times\left[-G_{1}, G_{1}\right]}|f(t, x, y)| .
\end{aligned}
$$

Theorem 11. Suppose $f:[0,1] \times R^{2} \rightarrow R$ is continuous. If there exist four constants $L_{i}, i=1,2,3,4, L_{2}>L_{1} \geq B$ and $L_{3}<L_{4} \leq B$, such that

$$
\begin{aligned}
& f(t, x, y) \geq 0, \quad \text { for }(t, x, y) \in[0,1] \times R \times\left[L_{1}, L_{2}\right], \\
& f(t, x, y) \leq 0, \quad \text { for }(t, x, y) \in[0,1] \times R \times\left[L_{3}, L_{4}\right],
\end{aligned}
$$

then Problem (3)-(4) has a solution $x \in C^{\alpha}[0,1]$ such that

$$
\begin{array}{r}
|x(t)| \leq G_{2}, \\
\left|D^{\alpha-1} x(t)\right| \leq G_{1}, \\
\left|D^{\alpha} x(t)\right| \leq G_{3},
\end{array}
$$

for $t \in[0,1]$.

Proof. Consider the family of boundary value problems

$$
\begin{aligned}
D^{\alpha} x(t) & =\lambda f\left(t, x(t), D^{\alpha-1} x(t)\right), \\
t \in[0,1], \lambda \in[0,1], & \\
x(0) & =A, \\
D^{\alpha-1} x(1) & =B .
\end{aligned}
$$

Denote

$$
\begin{aligned}
C_{B}^{\alpha} & {[0,1] } \\
& =\left\{x \in C^{\alpha}[0,1] \mid x(0)=A, D^{\alpha-1} x(1)=B\right\} .
\end{aligned}
$$

Define an operator $T_{\lambda}: C_{B}^{\alpha}[0,1] \rightarrow C_{B}^{\alpha}[0,1]$ as

$$
\begin{aligned}
\left(T_{\lambda} x\right)(t)= & \lambda \int_{0}^{1} G(t, s) f\left(s, x(s), D^{\alpha-1} x(s)\right) d s \\
& +\psi(t)
\end{aligned}
$$

where

$$
\begin{aligned}
& G(t, s)= \begin{cases}s \cdot s^{\alpha-2}, & 0 \leq s \leq t \leq 1 \\
t \cdot s^{\alpha-2}, & 0 \leq t \leq s \leq 1,\end{cases} \\
& \psi(t)=A+B t .
\end{aligned}
$$

Then standard arguments yield that $T_{\lambda}: C_{B}^{\alpha}[0,1] \rightarrow C_{B}^{\alpha}[0,1]$ is completely continuous. Clearly, the fixed point of operator $T_{\lambda}$ is the solution for Problem (22)-(23). 
Given $M>0$, let

$U=\left\{u \in C_{B}^{\alpha}[0,1]\left|\|u\|_{\alpha}<M+\right| A|+2| B \mid+1\right\}$.

We will prove that, for some positive number $M$, the map $T_{\lambda}$ : $\bar{U} \rightarrow C_{B}^{\alpha}[0,1]$ is admissible.

We claim that all the possible solutions of Problem (22)(23) on $C^{\alpha}[0,1]$ have a priori bound without dependence on $\lambda \in[0,1]$. Assume that the sets

$$
\begin{aligned}
& E_{0}=\left\{t \in[0,1] \mid L_{1}<D^{\alpha-1} x(t) \leq L_{2}\right\}, \\
& E_{1}=\left\{t \in[0,1] \mid L_{3} \leq D^{\alpha-1} x(t)<L_{4}\right\}
\end{aligned}
$$

are not empty.

Choose $t_{0} \in E_{0}$. Assume there exists $t_{0}^{\prime} \in\left(t_{0}, 1\right]$ such that

$$
D^{\alpha-1} x\left(t_{0}^{\prime}\right)<D^{\alpha-1} x\left(t_{0}\right) \text {. }
$$

Taking into account the fact that $D^{\alpha-1} x(t)$ is continuous, we can take $t_{0}^{\prime} \in\left(t_{0}, 1\right] \cap E_{0}$. The assumption on $f$ yields that $D^{\alpha} x(t)=\lambda f\left(t, x(t)\right.$ and $\left.D^{\alpha-1} x(t)\right) \geq 0$ for $t \in E_{0}$. Consequently, $D^{\alpha-1} x(t)$ is increasing on $E_{0}$. Thus

$$
D^{\alpha-1} x\left(t_{0}^{\prime}\right) \geq D^{\alpha-1} x\left(t_{0}\right),
$$

which is a contradiction to (29). So, there holds

$$
D^{\alpha-1} x(t) \geq D^{\alpha-1} x\left(t_{0}\right), \quad \text { for } t \in\left(t_{0}, 1\right],
$$

and, in particular,

$$
D^{\alpha-1} x(1) \geq D^{\alpha-1} x\left(t_{0}\right)>L_{1} \geq B,
$$

a contradiction to (23). This shows that $E_{0}=\varnothing$. The similar arguments can show that $E_{1}=\varnothing$.

By $D^{\alpha-1} x(1)=B$ and $D^{\alpha-1} x(t) \in C[0,1]$, there holds $L_{4} \leq D^{\alpha-1} x(t) \leq L_{1}$ for $t \in[0,1]$, and then $\left|D^{\alpha-1} x(t)\right| \leq G_{1}$. On the other hand, by Lemma 5 , for each $t \in(0,1]$, there exists $d \in(0, t)$ such that

$$
x(t)-x(0)=D^{\alpha-1} x(d) \cdot \frac{1}{\alpha-1} t^{\alpha-1} .
$$

Thus, $|x(t)| \leq G_{2}$. Finally (22) together with the continuity of $f$ and a priori estimations of $D^{\alpha-1} x(t)$ and $x(t)$ show that $\left|D^{\alpha} x(t)\right| \leq G_{3}$.

Set $M=G_{1}+G_{2}+G_{3}$, and one has

$$
\begin{aligned}
\|x(t)\|_{\alpha} & =\left\|D^{\alpha} x(t)\right\|_{0}+\left\|D^{\alpha-1} x(t)\right\|_{0}+\|x(t)\|_{0}<M \\
& <\infty .
\end{aligned}
$$

The above results indicate that $T_{\lambda}$ is completely continuous and has no fixed point on $\partial U$ and $T_{\lambda}$ is admissible on $\bar{U}$ for each $\lambda \in[0,1]$. By Lemma 7 , the constant map $T_{0}(x) \equiv \psi(t)$ for $x \in \bar{U}$ is essential. Combined with Lemma 9, the topological transversality theorem, $T_{1}$, is essential. So
Problem (3)-(4) has a solution $x \in \bar{U}$. Moreover, the above arguments show that the solution $x$ satisfies

$$
\begin{aligned}
|x(t)| & \leq G_{2}, \\
\left|D^{\alpha-1} x(t)\right| & \leq G_{1}, \\
\left|D^{\alpha} x(t)\right| & \leq G_{3},
\end{aligned}
$$

for $t \in[0,1]$.

The proof is completed.

The next theorem can be proved by similar arguments.

Theorem 12. Let $f:[0,1] \times R^{2} \rightarrow R$ be continuous. Suppose there exist four constants $L_{i}, i=1,2,3,4$, such that $L_{2}>L_{1} \geq$ $A$ and $L_{3}<L_{4} \leq A$ :

$$
\begin{aligned}
& f(t, x, y) \leq 0, \quad \text { for }(t, x, y) \in[0,1] \times R \times\left[L_{1}, L_{2}\right], \\
& f(t, x, y) \geq 0, \quad \text { for }(t, x, y) \in[0,1] \times R \times\left[L_{3}, L_{4}\right] .
\end{aligned}
$$

Then Problem (3)-(4) has a solution $x \in C^{\alpha}[0,1]$ such that

$$
\begin{aligned}
|x(t)| & \leq G_{2}, \\
\left|D^{\alpha-1} x(t)\right| & \leq G_{1}, \\
\left|D^{\alpha} x(t)\right| & \leq G_{3},
\end{aligned}
$$

for $t \in[0,1]$.

Example 13. Consider the following fractional BVPs:

$$
\begin{aligned}
D^{3 / 2} x(t)= & \sin x(t)+\left[D^{1 / 2} x(t)\right]^{5}-\frac{5}{2}\left[D^{1 / 2} x(t)\right]^{4} \\
& +\frac{3}{2}\left[D^{1 / 2} x(t)\right]^{3}, \\
x(0)= & 0 \\
D^{1 / 2} x(1)= & 1 .
\end{aligned}
$$

Let $L_{1}=2, L_{2}=4, L_{3}=-2$, and $L_{4}=-1$, and then the simple computation show that $G_{1}=4, G_{2}=8, G_{3}=1.08252$, and

$$
\begin{aligned}
& f(t, x, y) \geq 0, \quad \text { for }(t, x, y) \in[0,1] \times R \times[2,4], \\
& f(t, x, y) \leq 0, \quad \text { for }(t, x, y) \in[0,1] \times R \times[-2,-1] .
\end{aligned}
$$

By the use of Theorem 11, Problem (38) has a solution $x \in$ $C^{3 / 2}[0,1]$ such that

$$
\begin{aligned}
|x(t)| & \leq 8, \\
\left|D^{1 / 2} x(t)\right| & \leq 4, \\
\left|D^{3 / 2} x(t)\right| & \leq 1.08252,
\end{aligned}
$$

for $t \in[0,1]$. 


\section{Conclusion}

By the use of the topological transversality theorem, some existence results for a class of fractional differential equations with certain boundary value conditions are obtained. The main condition is sign condition which is easy to be satisfied and checked.

\section{Conflicts of Interest}

The authors declare that there are no conflicts of interest regarding the publication of this paper.

\section{Acknowledgments}

This work is supported by NSFC (11571207), the Taishan Scholar Project, and SDUST Graduate Innovation Project SDKDYC170343.

\section{References}

[1] S. Zhang, "The existence of a positive solution for a nonlinear fractional differential equation," Journal of Mathematical Analysis and Applications, vol. 252, no. 2, pp. 804-812, 2000.

[2] Z. Bai, "On positive solutions of a nonlocal fractional boundary value problem," Nonlinear Analysis. Theory, Methods \& Applications. An International Multidisciplinary Journal, vol. 72, no. 2, pp. 916-924, 2010.

[3] A. Atangana, "A novel model for the lassa hemorrhagic fever: deathly disease for pregnant women," Neural Computing and Applications, vol. 26, no. 8, pp. 1895-1903, 2015.

[4] Z. Bai, X. Dong, and C. Yin, "Existence results for impulsive nonlinear fractional differential equation with mixed boundary conditions," Boundary Value Problems, Paper No. 63, 11 pages, 2016.

[5] Z. Bai, S. Zhang, S. Sun, and C. Yin, "Monotone iterative method for a class of fractional differential equations," Electronic Journal of Differential Equations, vol. 6, pp. 1-8, 2016.

[6] Z. Bai and Y. Zhang, "Solvability of fractional three-point boundary value problems with nonlinear growth," Applied Mathematics and Computation, vol. 218, no. 5, pp. 1719-1725, 2011.

[7] Z. Bai and Y. Zhang, "The existence of solutions for a fractional multi-point boundary value problem," Computers \& Mathematics with Applications, vol. 60, no. 8, pp. 2364-2372, 2010.

[8] Z. B. Bai and T. T. Qiu, "Existence of positive solution for singular fractional differential equation," Applied Mathematics and Computation, vol. 215, no. 7, pp. 2761-2767, 2009.

[9] Y. Cui, "Uniqueness of solution for boundary value problems for fractional differential equations," Applied Mathematics Letters, vol. 51, pp. 48-54, 2016.

[10] H. H. Dong, B. Y. Guo, and B. S. Yin, "Generalized fractional supertrace identity for Hamiltonian structure of NLS-MKdV hierarchy with self-consistent sources," Analysis and Mathematical Physics, vol. 6, no. 2, pp. 199-209, 2016.

[11] X. Dong, Z. Bai, and S. Zhang, "Positive solutions to boundary value problems of $p$-Laplacian with fractional derivative," Boundary Value Problems, Paper No. 5, 15 pages, 2017.

[12] X. Dong, Z. Bai, and W. Zhang, "Positive solutions for nonlinear eigenvalue problems with conformable fractional differential derivatives," Journal of Shandong University of Science and Technology (Natural Science), vol. 35, pp. 85-90, 2016 (Chinese).

[13] Y. Hua and X. Yu, "On the ground state solution for a critical fractional Laplacian equation," Nonlinear Analysis: Theory, Methods \& Applications, vol. 87, pp. 116-125, 2013.

[14] U. Katugampola, "A new fractional derivative with classical properties," Mathematics, vol. 6, no. 4, pp. 1-15, 2014.

[15] S. Liu, A. Debbouche, and J. Wang, "On the iterative learning control for stochastic impulsive differential equations with randomly varying trial lengths," Journal of Computational and Applied Mathematics, vol. 312, no. 1, pp. 47-57, 2017.

[16] X. Liu, M. Jia, and W. Ge, "The method of lower and upper solutions for mixed fractional four-point boundary value problem with $p$-Laplacian operator," Applied Mathematics Letters, vol. 65, pp. 56-62, 2017.

[17] X. Liu, M. Jia, and B. Wu, "Existence and uniqueness of solution for fractional differential equations with integral boundary conditions," Electronic Journal of Qualitative Theory of Differential Equations, pp. 1-10, 2009.

[18] X. Meng, S. Zhao, T. Feng, and T. Zhang, "Dynamics of a novel nonlinear stochastic SIS epidemic model with double epidemic hypothesis," Journal of Mathematical Analysis and Applications, vol. 433, no. 1, pp. 227-242, 2016.

[19] X. Shang and Z. Du, "Traveling waves in a generalized nonlinear dispersive-dissipative equation," Mathematical Methods in the Applied Sciences, vol. 39, no. 11, pp. 3035-3042, 2016.

[20] Q. Song, X. Dong, Z. Bai, and B. Chen, "Existence for fractional Dirichlet boundary value problem under barrier strip conditions," The Journal of Nonlinear Science and Applications, vol. 10, pp. 3592-3598, 2017.

[21] F. Wang, B. Chen, C. Lin, and X. Li, "Distributed adaptive neural control for stochastic nonlinear multi-agent systems," IEEE Transaction on cybernetics, vol. 47, no. 7, pp. 1795-1803, 2017.

[22] G. Wang, B. Ahmad, L. Zhang, and J. J. Nieto, "Comments on the concept of existence of solution for impulsive fractional differential equations," Communications in Nonlinear Science and Numerical Simulation, vol. 19, no. 3, pp. 401-403, 2014.

[23] J. Wang, Y. Zhou, and M. Feckan, "On recent developments in the theory of boundary value problems for impulsive fractional differential equations," Computers \& Mathematics with Applications, vol. 64, no. 10, pp. 3008-3020, 2012.

[24] Z. Wang, "A numerical method for delayed fractional-order differential equations," Journal of Applied Mathematics, Article ID 256071, 7 pages, 2013.

[25] Z. Wang, X. Huang, and J. Zhou, "A numerical method for delayed fractional-order differential equations: based on G-L definition," Applied Mathematics \& Information Sciences, vol. 7, no. 2, pp. 525-529, 2013.

[26] Y. Xu, Z. Du, and L. Wei, "Geometric singular perturbation method to the existence and asymptotic behavior of traveling waves for a generalized Burgers-KdV equation," Nonlinear Dynamics, vol. 83, no. 1-2, pp. 65-73, 2016.

[27] S. Yan, J. Yang, and X. Yu, "Equations involving fractional Laplacian operator: compactness and application," Journal of Functional Analysis, vol. 269, no. 1, pp. 47-79, 2015.

[28] X. Yu, "Solutions of fractional Laplacian equations and their Morse indices," Journal of Differential Equations, vol. 260, no. 1, pp. 860-871, 2016.

[29] W. Zhang, Z. Bai, and S. Sun, "Extremal solutions for some periodic fractional differential equations," Advances in Difference Equations, Paper No. 179, 8 pages, 2016. 
[30] Y. Zou and Y. Cui, "Existence results for a functional boundary value problem of fractional differential equations," Advances in Difference Equations, 2013:233, 25 pages, 2013.

[31] P. Kelevedjiev, "Existence of solutions for two-point boundary value problems," Nonlinear Analysis: Theory, Methods \& Applications, vol. 22, no. 2, pp. 217-224, 1994.

[32] P. S. Kelevedjiev and S. Tersian, "Singular and nonsingular firstorder initial value problems," Journal of Mathematical Analysis and Applications, vol. 366, no. 2, pp. 516-524, 2010.

[33] A. Granas, R. B. Guenther, and J. W. Lee, "Applications of topological transversality to differential equations. I. Some nonlinear diffusion problems," Pacific Journal of Mathematics, vol. 89, no. 1, pp. 53-67, 1980. 


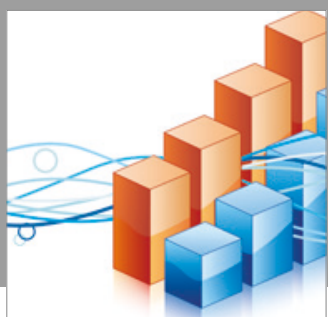

Advances in

Operations Research

vatersals

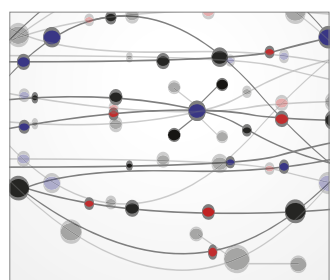

\section{The Scientific} World Journal
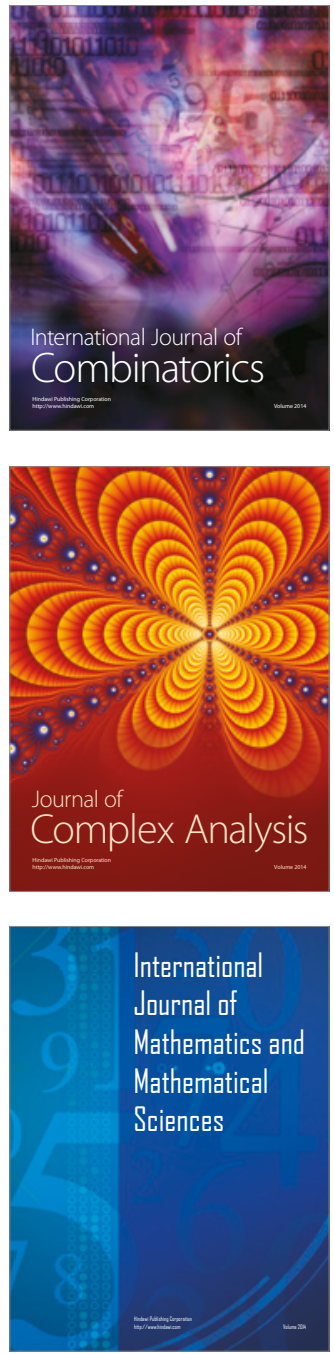
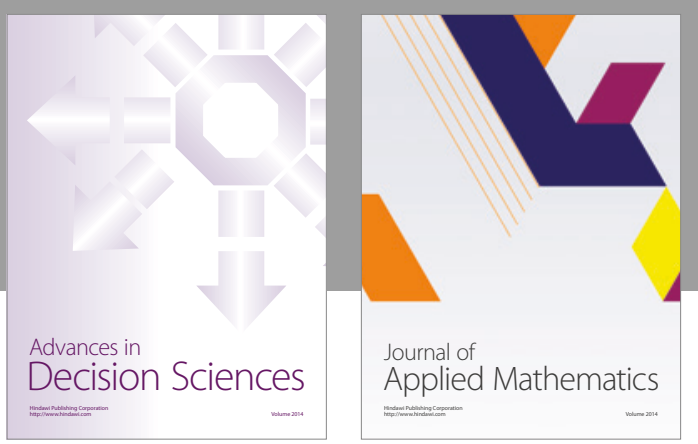

Algebra

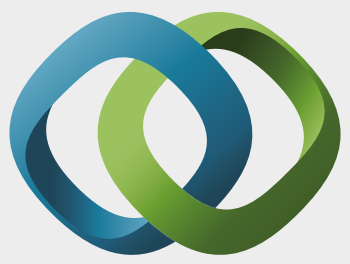

\section{Hindawi}

Submit your manuscripts at

https://www.hindawi.com
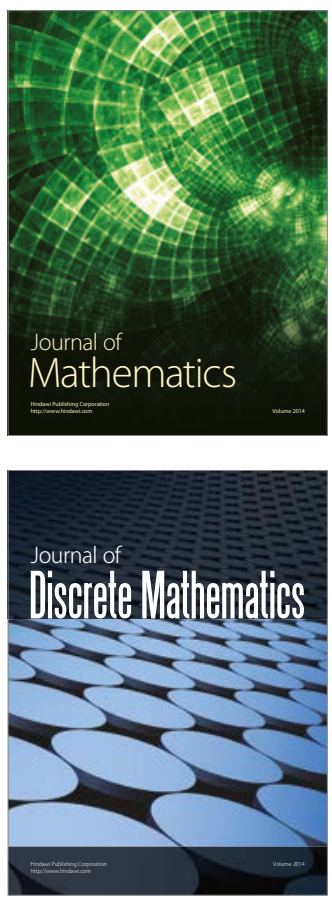

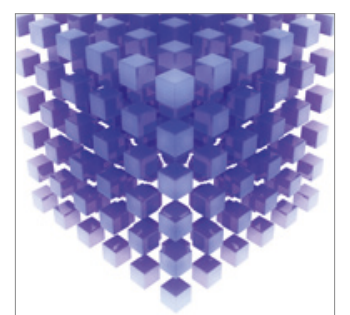

Mathematical Problems in Engineering
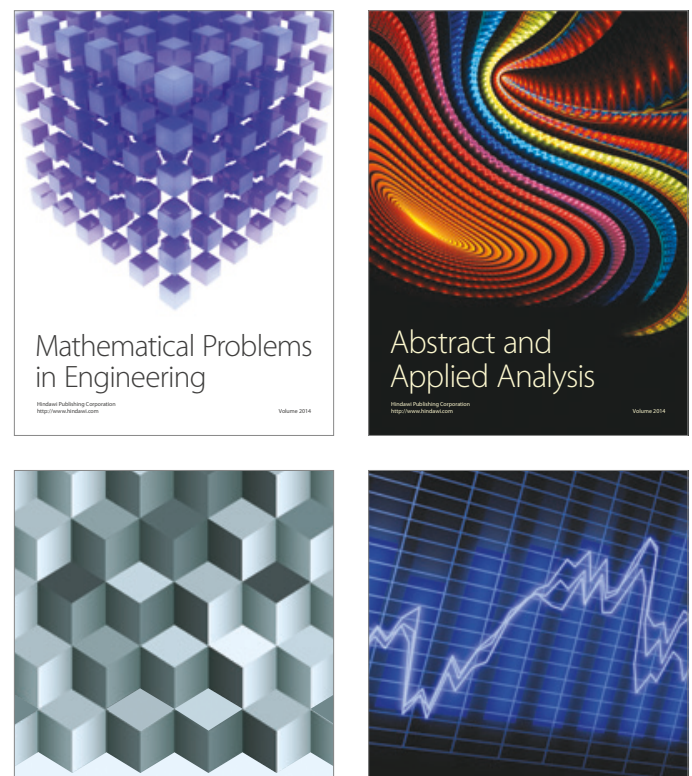

Journal of

Function Spaces

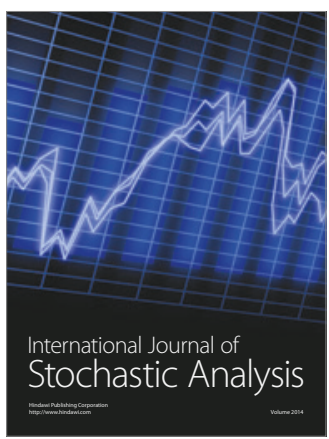

Probability and Statistics
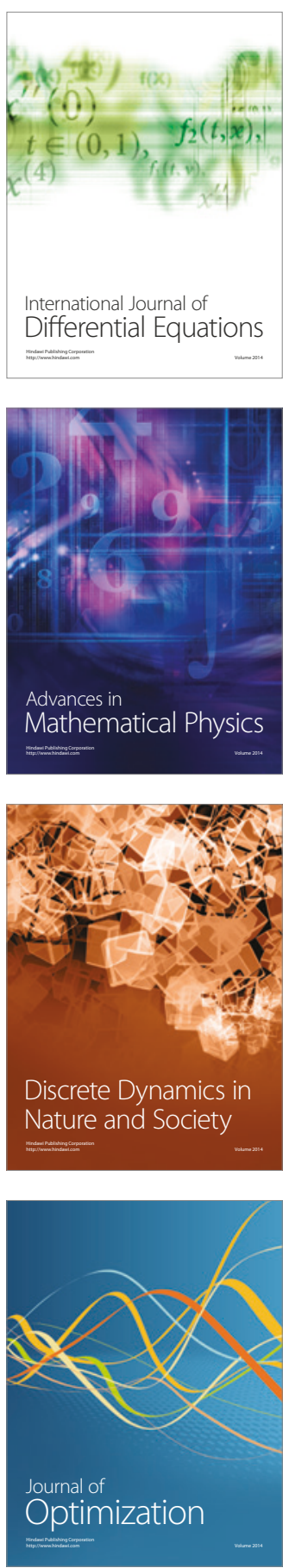